\title{
Efecto Protector de Croton palanostigma y Aloe vera frente a Injuria Aguda de Mucosa Gástrica inducida por Etanol en Ratas
}

\author{
SALOMÓN AYALA', DAVID DÍAZ', MANUEL PALOMINO', SEGUNDO ARMAS', JUAN PAZ3 \\ 'Departamento de Ciencias Dinámicas e Instituto de Patología, Facultad de Medicina UNMSM. \\ ${ }^{3}$ Estudiante del $5^{\circ}$ Año de la Escuela de Medicina Humana, UNMSM.
}

\begin{abstract}
RESUMEN
OBJETIVOS: Evaluar el grado de protección frente a la necrosis de la mucosa gástrica inducida por etanol con Croton palanostigma (Sangre de Grado) y Aloe vera, y compararlo con el de sucralfato y una suspensión de antiácido $\left(\mathrm{AlOH}_{3}+\mathrm{MgOH}_{3}+\right.$ simeticona), en un modelo experimental estandarizado en ratas. MATERIAL Y MÉTODOS: Se evaluó a 56 animales, administrándoles en ayunas uno de 7 pretratamientos: solución salina, C. palanostigma $(0,460,8 \mathrm{~mL} / \mathrm{kg})$, A. vera $(7,5$ ó $3,2 \mathrm{~mL} / \mathrm{kg})$, sucralfato $(500 \mathrm{mg} / \mathrm{kg})$ o antiácido. Una hora más tarde, se administró por vía intragástrica $2 \mathrm{~mL}$ de etanol al $100 \%$. Se realizó una evaluación cualitativa y cuantitativa, macroscópica y microscópica, de las lesiones gástricas. RESULTADOS: El pretratamiento con C. palanostigma redujo significativamente la aparición de necrosis hemorrágica inducida por etanol, $A$. vera no presentó efecto protector, mientras que sí lo presentaron el sucralfato, en grado significativo, y el antiacido, en menor grado. CONCLUSIÓN: En nuestras condiciones experimentales, Croton palanostigma presentó un importante efecto protector y potencial terapéutico.
\end{abstract}

Palabras claves: Mucosa Gástrica; Plantas Medicinales; Agentes Antiulcerosos; Modelos Animales de Enfermedad.

PROTECTIVE EFFECT OF Croton palanostigma, Aloe vera, SUCRALFATE AND ANTACID AGAINST ACUTE ETHANOL-INDUCED GASTRIC MUCOSA INJURY

SUMMARY

OBJECTIVE: The gastric protective abilities of Croton palanostigma (dragonis blood), Aloe vera, sucralfate, and antacid against acute ethanol-induced necrosis by means of a standard experimental model in rats, were compared. MATERIAL AND METHODS: A pretreatment with either saline solution; $0,8 \mathrm{~mL} / \mathrm{kg}$ or $0,4 \mathrm{~mL} / \mathrm{kg}$ C. palanostigma; $7,5 \mathrm{~mL} / \mathrm{kg}$ or $3,2 \mathrm{~mL} / \mathrm{kg}$ A. vera; $500 \mathrm{mg} / \mathrm{kg}$ sucralfate; or antacid was given to 56 fasted rats. Then we accomplished an intragastric administration of $2 \mathrm{~mL}$ of $100 \%$ ethanol one hour later. A gastrectomy was performed four hours later followed by a macroscopic and histologycal evaluation of the gastric lesions. RESULTS: $C$. palanostigma pretreatment resulted in a significant reduction of ethanol-induced gastric mucosa necrosis. A. vera failed to show mucosal protection. Sucralfate showed a significant protective effect, while antacid exhibited a lesser protective effect. CONCLUSION: In the present study, C. palanostigma showed an important protective effect, being potentially useful as a therapeutic tool.

Key words: Gastric Mucosa; Plants, Medicinal; Anti-Ulcer Agents; Disease Models, Animal.

\footnotetext{
Correspondencia:

Dr. Salomón Ayala Pío

Faculiad de Medicina - UNMSM.

Av. Grau 755 - Lima L. Perú.

E-mail:dl00032@sanfer.unmsm.edu.pe
} 


\section{INTRODUCCIÓN}

Las gastritis, úlceras pépticas y en general las manifestaciones ácido pépticas del tubo digestivo alto, y particularmente del estómago, son frecuentes en nuestra población, usándose para el tratamiento diversos medicamentos con mayor o menor eficacia. En la medicina tradicional se ha reportado la acción antiinflamatoria y cicatrizante de úlcera péptica de varios productos vegetales, los cuales son usados por la población general como recursos terapéuticos por su fácil obtención y costo económico, refiriendose mínimas reacciones adversas $\left({ }^{1-3}\right)$. Se ha estudiado el plátano (Musa sapientum L. musaceae) en varios modelos experimentales ( ${ }^{4.5}$ ), así como la col (Brassica oleracea L. cruciferae), la cual usada como antiulceroso llevó al desarrollo del gafarnate $\left({ }^{6}\right)$. Sin embargo, en nuestro medio existen pocos trabajos experimentales que corroboren los supuestos beneficios de diversas plantas, incluyendo Croton palanostigma (Sangre de Grado) y Aloe vera $\left({ }^{7.8}\right)$.

Existen diversos modelos experimentales para abordar el estudio de productos con potencial eficacia antiulcerosa. El etanol absoluto, empleado como Eccurso experimental por ser una sustancia gastrolesiva, produce daño de la mucosa por mecanismos independientes a Ia acidez gástrica, factor importante en la ulcerogénesis $\left({ }^{8-10}\right)$. Esta característica permite evaluar el denominado efecto citoprotector propuesto para varios grupos de fármacos usados en las gastropatías y úlcera péptica, incluyendo sucralfato $\left({ }^{11}\right)$, carbenoxolona $\left({ }^{12}\right)$ y una suspensión de antiácido [ver material y métodos $\left({ }^{13.14}\right)$ ]. El presente estudio tuvo como objetivo evaluar y comparar el efecto citoprotector de Croton palanostigma, Aloe vera, sucralfato y antiácido en la mucosa gástrica lesionada con etanol absoluto.

\section{MÁTERIAL Y MÉTODOS}

\section{Material vegetal}

La resina de Croton palanostigma Klotzch, denominado tradicionalmente "Sangre de Grado", familia Euphorbiaceae, fue obtenida tres meses antes del experimento de la selva del departamento de San Martín (Perú) y se empleó en su estado natural líquido, y conservada en frasco de vidrio esterril a $10^{\circ} \mathrm{C}$. Las hojas de Aloe vera $L$. fueron recolectadas un día antes del experimento, se licuó para disminuir su consistencia y faci- litar su administración. En ambos casos el material fue colectado por un etnobotânico.

\section{Material farmacológico}

Se empleo una suspensión de antiácido (Mylanta $\mathrm{II}^{\circledR}$ suspensión, Parke Davis) con la siguiente composición: hidróxido de magnesio $400 \mathrm{mg}$, hidróxido de aluminio $400 \mathrm{mg}$, simeticona $30 \mathrm{mg}$, sodio $1,14 \mathrm{mg}$ (cada $5 \mathrm{~mL}$ ); a la que en adelante nos referiremos como "antiácido". Iguamente se empleó una suspensión de sucralfato (Ulcogant ${ }^{\oplus}$, Merck Sharp \& Dohme) que contiene $1 \mathrm{~g}$ en $5 \mathrm{~mL}$. Los animales fueron anestesiados con pentobarbital sódico (Halatal ${ }^{\oplus}$, Sanivet), que contiene $6,5 \mathrm{~g}$ en vehículo y exipientes c.s.p. $100 \mathrm{~mL}$.

\section{Métodos}

El estudio se realizó con 56 ratas albinas de la cepa Balck, de ambos sexos, con un rango de peso de 200 a $250 \mathrm{~g}$, obtenidas del Instituto Nacional de Salud. Estos animales fueron divididos aleatoriamente en 7 grupos de ocho ratas cada uno y trabajados grupalmente en fechas diferentes. Todos los animales fueron puestos en jaulas individuales y en ayunas $24 \mathrm{~h}$ antes del experimento. A cada grupo se les administró antes de la injuria aguda uno de los siguientes pretratamientos por vía orogástrica a través de una cánula metálica:

1. Solución salina (CINa 0,9\%) [control]

2. Croton palanostigma

3. Croton palanostigma

4. Aloevera

5. Aloe vera

6. Antiácido (Mylanta $I^{\oplus}$ suspensión)

7. Sucralfato suspensión

$2,0 \mathrm{~mL}$ $0,8 \mathrm{~mL} / \mathrm{kg}$ $0,4 \mathrm{~mL} / \mathrm{kg}$ $7,5 \mathrm{~mL} / \mathrm{kg}$ $3,2 \mathrm{~mL} / \mathrm{kg}$ $2,0 \mathrm{~mL}$ $500 \mathrm{mg} / \mathrm{kg}$

El volumen administrado fue $2 \mathrm{~mL}$, completándose en caso necesario con solución salina fisiológica. Una hora después del pretratamiento se administró $2 \mathrm{~mL}$ de etanol absoluto a todas las ratas vía orogástrica. Cuatro horas después de la injuria con etanol, las ratas fueron anestesiadas con pentobarbital sódico ( $50 \mathrm{mg} / \mathrm{kg}$ de peso, vía intraperitoneal) y se les practicó una laparotomía seguida de gastrectomía. El estómago fue abierto por la curvatura mayor, lavado con solución salina, de inmediato extendido totalmente sobre una base plana, con la mucosa gástrica hacia arriba y fotografiado en fresco. La fijación se hizo en formol neutro al $10 \%$ por lapso de una semana. A todos los estómagos fijados se les tomó copias fotostáticas (se utilizaron máquinas fotocopiadoras Xerox) por la cara que corresponde a la 
mucosa, tamaño natural, con el propósito de encontrar el porcentaje de área lesionada de la mucosa gástrica de cada estómago. Se trabajó cada copia fotostática comparándola con su respectivo órgano fijado para retocarlas cuidadosamente, con el fin de hacer resaltar sólo las lesiones ubicadas en la mucosa glandular. Las copias fotostáticas retocadas fueron nuevamente fotocopiadas a una ampliación de $100 \%$. Se recortó cuidadosamente cada copia ampliada por todo el perímetro correspondiente al área de mucosa glandular y se pesó el papel recortado de dicha área en una balanza analítica, cortando nuevamente la totalidad de las áreas marcadas como lesiones de la mucosa y pesadas en la misma balanza.

Se determinó en cada estómago estudiado, el porcentaje de la mucosa lesionada con respecto al área total de la mucosa glandular, considerándose la relación porcentual del peso como representativa de las áreas, con la siguiente fórmula matemática:

Qo de área lesionada $=\frac{\text { peso del total de lesiones } \times 100}{\text { peso de la mucosa total }}$

A partir de esta área se determinó el promedio y la desviación estándar del porcentaje de mucosa lesionada para cada grupo.

Para la evaluación microscópica se analizaron los estómagos fijados, se tomó muestras de todas las lesiones de la mucosa gástrica y se las representó en las copias xerox correspondientes (Figura $\mathrm{N}^{\circ} 1$ ). Las muestras se embebieron en parafina y se tomaron cortes micrométricos de 4 a 5 micras, teñidos con hematoxilina-eosina y alcian blue más ácido peryódico de Schiff.

El estudio de la profundidad (micras) de las lesiones necrohemorrágicas de la mucosa gástrica, se determinó con un lente ocular con regla micrométrica.

La evaluación estadística se realizó con los métodos de análisis de varianza y la prueba de $t$ de Student, aceptándose significación estadística para un valor de $p \leq 0,05$.

\section{RESULTADOS}

De acuerdo a la evaluación macroscópica cualitativa, el grupo control que recibió solución salina presen-

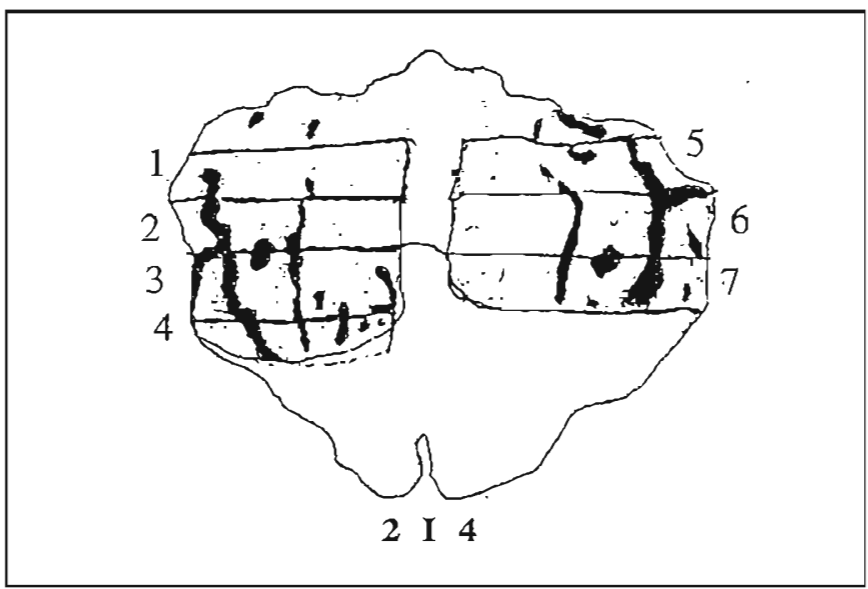

Fig. $\mathbf{N}^{\circ}$ 1.- Esquema del mapeo de la muestra de estómago fijado para el estudio microscópico de las lesiones. Se observa siete áreas de corte.

tó el mayor grado de lesiones y enrojecimiento de la mucosa gástrica, mientras que el tratado con $C$. palanostigma $(0,4 \mathrm{~mL} / \mathrm{kg})$ evidenció el menor grado, similar a lo evidenciado con sucralfato. El grupo tratado con $A$. vera $3,2 \mathrm{~mL} / \mathrm{kg}$ presentó lesiones en grado semejante al grupo control que recibió solución salina (Tabla $N^{\circ} 1$ y Figura $N^{\circ} 2$ ).

En la evaluación macroscópica cuantitativa, las lesiones en el pretratamiento con solución salina alcanzaron un promedio de $15,47 \% \pm 9,50 \%$ del total del área glandular del estómago, superior al efecto obtenido con C. palanostigma (a ambas dosis), con el que se encontró una reducción altamente significativa de las áreas afectadas. A. vera a la dosis mayor presentó un $7,07 \% \pm 5,17 \%$, dando una protección intermedia, aunque estadísticamente significativa $(p<0,025)$, no evidenciada con la dosis menor $(p<0,25)$.

En el caso del antiácido, el área lesionada alcanzó una reducción estađísticamente significativa en la formación de lesiones, igual que con sucralfato, aunque este último fármaco tuvo el mayor efecto protector en nuestras condiciones experimentales (Tabla $\mathrm{N}^{\circ} 2$ ).

La evaluación microscópica (Figura $\mathrm{N}^{\circ} 3$ ) mostró que la menor profundidad de las lesiones aparece en el grupo de estómagos pretratados con C. palanostigma $(0,4 \mathrm{~mL} / \mathrm{kg})$. Aloe vera $(3,2 \mathrm{~mL} / \mathrm{kg})$ también presentó menor profundidad de las lesiones. Los grupos trata- 
Tabla $N^{\circ}$ 1.- Grado de lesiones y de enrojecimiento inducidas por etanol en la evaluación macroscópica cualitativa, según los diferentes grupos de pretratamiento.

\begin{tabular}{lllllll}
\hline Lesiones & $\mathbf{0}$ & + & ++ & +++ & ++++ & $\begin{array}{c}\text { Total } \\
\mathbf{n}\end{array}$ \\
\hline Solución salina & 0 & 0 & 1 & 3 & 4 & 8 \\
C. palanostigma $0,8 \mathrm{~mL} / \mathrm{kg}$. & 3 & 4 & 1 & 0 & 0 & 8 \\
C. palanostigma $0,4 \mathrm{~mL} / \mathrm{kg}$. & 5 & 3 & 0 & 0 & 0 & 8 \\
Aloe vera $7,5 \mathrm{~mL} / \mathrm{kg}$. & 1 & 0 & 3 & 3 & 1 & 8 \\
Aloe vera 3,2 mL/kg. & 1 & 0 & 0 & 3 & 4 & 8 \\
Mylanta II & 1 & 1 & 2 & 3 & 1 & 8 \\
Sucralfato & 2 & 4 & 2 & 0 & 0 & 8 \\
\hline
\end{tabular}

dos con C. palanostigma $(0,8 \mathrm{~mL} / \mathrm{kg})$, antiácido y sucralfato presentaron menor profundidad de las lesiones necrohemorrágicas que el grupo control, aunque en niveles no estadísticamente significativos. Aloe vera $(7,5 \mathrm{~mL} / \mathrm{kg})$ mostró mayor profundidad que el grupo control, aunque tampoco estadísticamente significativa (Tabla $\mathrm{N}^{\circ} 3$ ).

\section{DISCUSIÓN}

Estudios anteriores han demostrado experimentalmente la propiedad cicatrizante de heridas y los efectos antiinflamatorios del C. palanostigma, C. lechleri y C. draconoides, tanto empleando la resina entera como una fracción alcaloide, denominada taspina $\left({ }^{15-17}\right)$, que

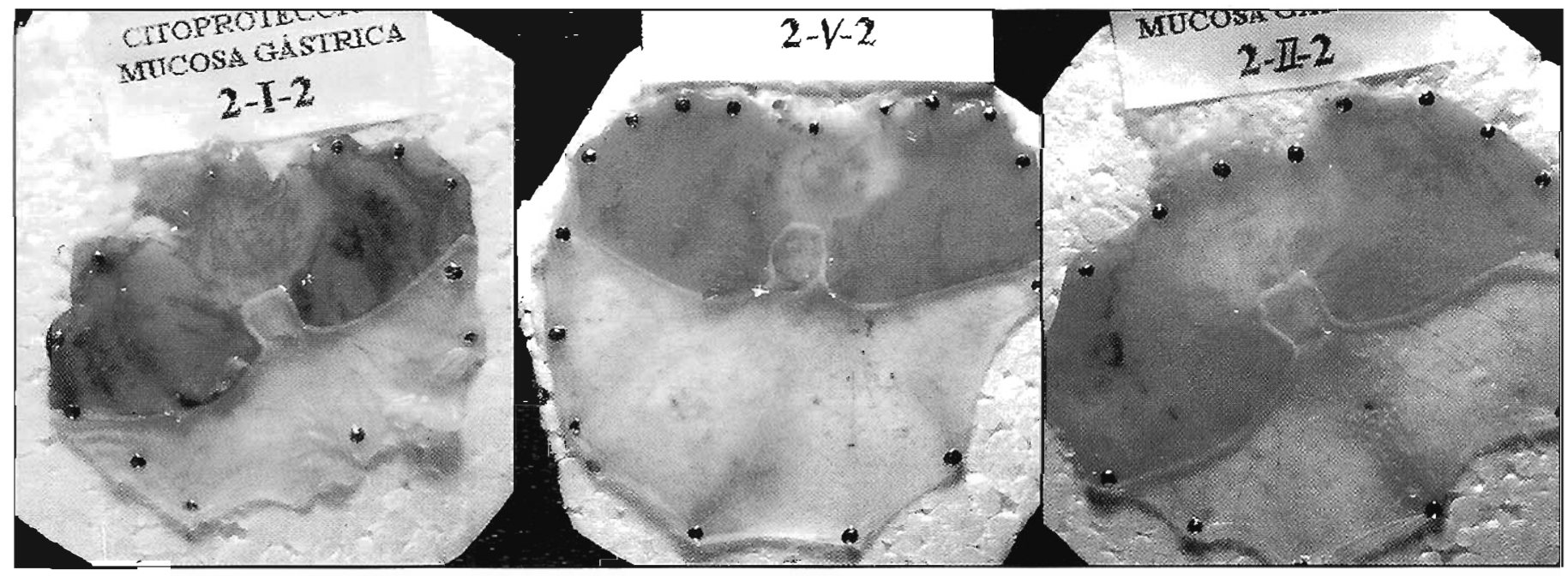

Fig. No 2.- Izquierda: aspecto macroscópico de la mucosa gástrica en rata control. Centro: estómago de rata que recibió pretratamiento con $C$. palanostigma. Derecha: estómago de rata que recibió pretratamiento con antiácido. Nótese las lesiones necrohemorrágicas francas en la rata pretratada con solución salina y la relativa ausencia de tales lesiones en los otros dos casos. 
Tabla $N^{\circ}$ 2.- Porcentaje de áreas necrohemorrágicas en la mucosa gástrica según la evaluación macroscópica cuantitativa.

\begin{tabular}{lccc}
\hline \multicolumn{1}{c}{ Pretratamiento } & $\begin{array}{c}\text { Número de } \\
\text { animales }\end{array}$ & $\begin{array}{c}\text { Porcentaje de áreas } \\
\text { necrohemorrágicas } \\
\text { (Promedio y DE) }\end{array}$ & $\begin{array}{c}\text { Valor de } \\
p^{*}\end{array}$ \\
\hline Solución salina & 8 & $15,47 \pm 9,50$ & \\
C. palanostigma $0,8 \mathrm{~mL} / \mathrm{kg}$ & 8 & $2,66 \pm 1,59$ & $<0,005$ \\
C. palanostigma $0,4 \mathrm{~mL} / \mathrm{kg}$ & 8 & $1,83 \pm 1,83$ & $<0,005$ \\
A. vera $7,5 \mathrm{~mL} / \mathrm{kg}$ & 8 & $7,07 \pm 5,17$ & $<0,025$ \\
A. vera 3,2 $\mathrm{mL} / \mathrm{kg}$ & 8 & $12,64 \pm 6,37$ & $<0,25$ \\
Mylanta $\mathrm{II}^{\otimes}$ & 8 & $7,10 \pm 5,39$ & $<0,005$ \\
Sucralfato & 8 & $1,73 \pm 1,07$ & $<0,0005$ \\
\hline
\end{tabular}

* Comparación con el grupo de solución salina usando t de Student.

parece ser un fitocomplejo responsable, en parte, de estas actividades. En el presente estudio demostramos el significativo efecto protector de mucosa gástrica de C. palanostigma en dosis de 0,8 y $0,4 \mathrm{~mL} / \mathrm{kg}$ administradas por vía oral en ratas. Estudios preliminares en nuestro medio muestran hallazgos similares $\left({ }^{7.8}\right)$.

Aunque la profundidad microscópica de las lesiones en el grupo tratado con C. palanostigma fue menor que la observada en el grupo control, las diferencias no fueron estadísticamente significativas. Es de notar que el mismo resultado se obtuvo con antiácido y sucralfato, fármacos de reconocido efecto citoprotector, de tal manera que consignamos los hallazgos macroscópicos como los resultados obtenidos más confiables.

Debido a que usamos el etanol como agente gastrolesivo, el cual produce daño independientemente de la acidez gástrica $\left({ }^{9.10}\right)$, el mecanismo de los fármacos protectores se puede catalogar como citoprotector. En nuestro estudio hemos observado macroscópicamente la adherencia pertinaz de la Sangre de Grado en las zonas necrohemorrágicas, la cual tuvo que ser retirada con lavados repetidos, y aún con fricción mecánica suave en algunos estómagos fijados. Además de esta "barrera protectora", como en el caso del sucralfato, los mecanismos de acción podrían ser múltiples y requieren mayores estudios.
En cuanto al potencial toxicológico del $C$. palanostigma, se ha observado que la toxicidad aguda de la resina v.o. es baja, siendo la $\mathrm{DL}_{50}$ en ratones $24 \mathrm{~mL} / \mathrm{kg}$, lo cual es 60 veces mayor que la dosis de $0,4 \mathrm{mg} / \mathrm{kg}$ según lo hallado por Ayala, Jurupe, Díaz y Espejo (comunicación personal) que se mostró eficaz en nuestro experimento. Igualmente, se requieren mayores estudios fitoquímicos y de toxiciđad subcrónica previos a su potencial evaluación clínica.

Con respecto a Aloe vera, no se ha encontrado efecto significativo en las evaluaciones macroscópicas. Sin embargo, 'Tapia y col. ( (') han reportado el efecto protector de esta planta. En el examen microscópico, los hallazgos de la profundidad de las lesiones según las dosis usadas parecen contradictorios: en la dosis de $3,2 \mathrm{~mL} / \mathrm{kg}$ es protector y a la dosis de $7,5 \mathrm{~mL} / \mathrm{kg}$ estadísticamente no se verifica este efecto. Estos datos no contribuyen a confirmar un efecto citoprotector.

El sucralfato y el antiácido redujeron significativamente la aparición de necrosis de la mucosa gástrica inducida por etanol. La capacidad protectora del sucralfato contra la injuria de la mucosa gástrica por varios agentes lesivos ha sido reportada previamente $\left({ }^{18.19}\right)$, aunque el mecanismo no ha sido claramente es tablecido, pudiendo incluir la formación de una "barrera protectora" en la mucosa lesionada, desactivación 
Tabla $N^{\circ}$ 3.- Profundidad de las lesiones necrohemorrágicas de la mucosa gástrica inducidas por etanol.

\begin{tabular}{lcc}
\hline \multicolumn{1}{c}{ Grupos } & $\begin{array}{c}\text { Profundidad en micras } \\
\text { (Promedio } \pm \text { DE) }\end{array}$ & $\begin{array}{c}\text { Valor de } \\
\boldsymbol{p}^{*}\end{array}$ \\
\hline Solución Salina & $166 \pm 84,85$ & \\
C. palanostigma $0,8 \mathrm{~mL} / \mathrm{kg}$ & $133 \pm 76,04$ & $<0,25$ \\
C. palanostigma $0,4 \mathrm{~mL} / \mathrm{kg}$ & $30 \pm 0$ & $<0,005$ \\
Aloevera $7,5 \mathrm{~mL} / \mathrm{kg}$ & $194 \pm 82,68$ & $>0,25$ \\
Aloevera $3,2 \mathrm{~mL} / \mathrm{kg}$ & $98 \pm 0,02$ & $<0,05$ \\
Suspensión de antiácido & $157 \pm 74,32$ & $<0,25$ \\
Sucralfato & $122 \pm 51,67$ & $<0,25$ \\
\hline
\end{tabular}

* Comparación con el grupo de Solución Salina utilizando i de Student.

y unión con pepsina, y ligazón con ácidos biliares ( ${ }^{20.21}$ ), eventos que podrían promover la cicatrización de la úlcera en el hombre $\left({ }^{22}\right)$. Estudios anteriores han demosrado que la liberación de prostaglandinas puede ser gualmente parte de este efecto $\left({ }^{22.23}\right)$. También en algunos modelos experimentales los cambios morfológicos, histológicos, ultraestructurales y funcio- nales son similares a los inducidos por prostaglandinas y el ácido araquidónico, precursor de prostaglandinas $\left({ }^{24.25}\right)$. Estas acciones pueden aplicarse a la mucosa gástrica humana ${ }^{26}$ ) y explicar no solamente el efecto terapéutico sino la eficacia profiláctica del sucralfato en la prevención de la recurrencia de la úlcera en humanos $\left({ }^{27.30}\right.$ ) y de la aparición de úlcera de estrés en

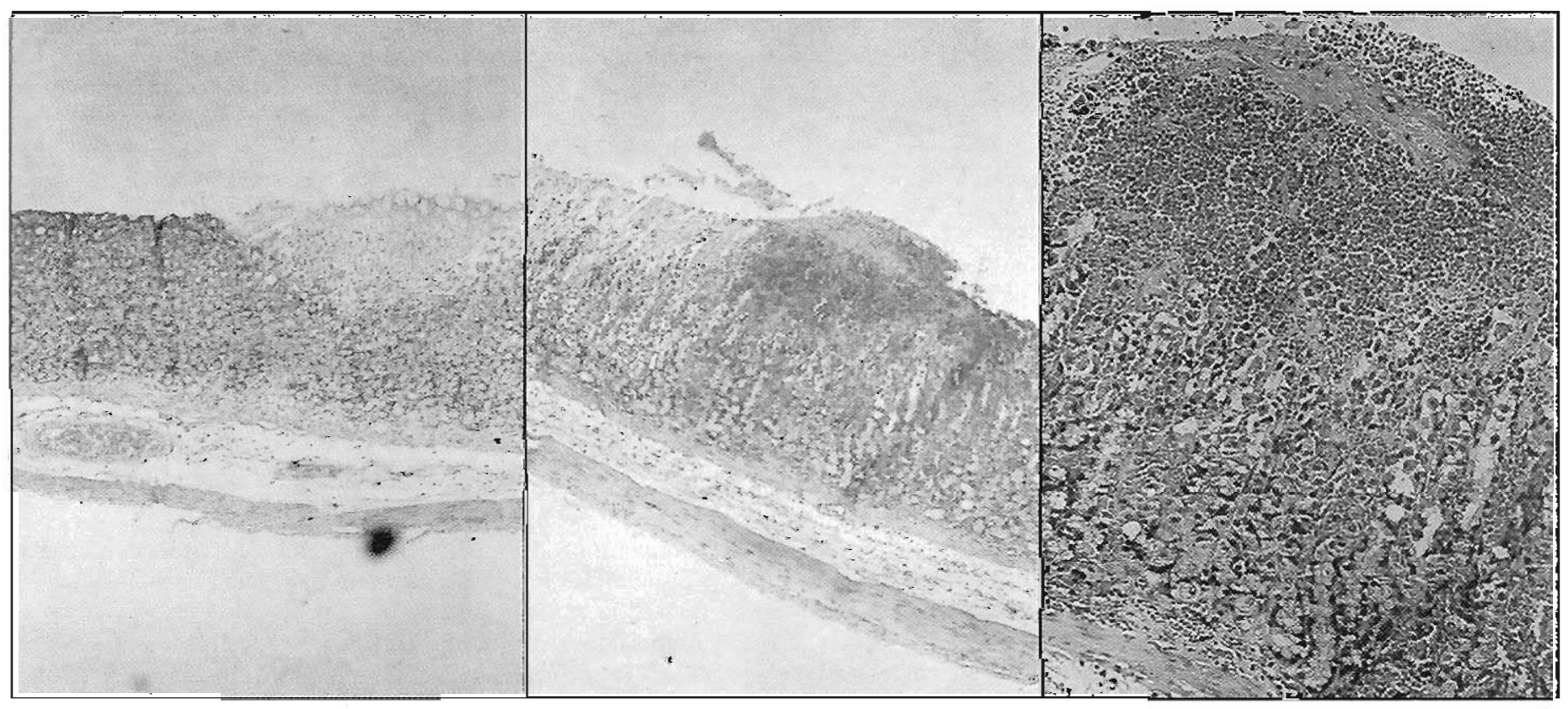

Fig. $N^{\circ}$ 3.- La microfotografía de la izquierda muestra lesión necrohemorrágica focal en mucosa con gran congestión vascular submucosa (H-E 10X), mientras que las lesiones del centro y la derecha son focales y profundas, y comprometen hasta muscularis mucosae, con edema y microhemoragia en submucosa (H-E 10X y 20X, respectivamente). 
pacientes gravemente enfermos ("1). El antiácido también protegió a la mucosa gástrica contra la injuria por etanol, pero la protección fue menor que la del sucralfato. Debido a que la injuria por etanol es independiente del ácido luminal, la acción tampón del antiácido no se considera responsable de su acción protectora. Tanto el antiácido como el sucralfato contienen aluminio insoluble, si el hidróxido de aluminio es activo en proteger la mucosa gástrica, se puede esperar que el antiácido y el sucralfato producirían similar efecto sobre ésta, ya que el antiácido y el hidróxido de aluminio tienen efectos sobre la mucosa gástrica normal que es similar a los del sucralfato, incluyendo incremento en la liberación de moco y prostaglandinas $\mathrm{E}_{2}\left({ }^{14}\right)$.

\section{CONCLUSIÓN}

En nuestras condiciones experimentales, Croton palanostigma presentó un importante efecto protector $y$ potencial eficacia terapéutica.

\section{AGRADECIMIENTOS}

Expresamos nuestro agradecimiento a la Dra. Hilda Jurupe Chico por revisar el manuscrito y al Sr. José Schunke V. por la recolección del material vegetal.

Este trabajo se realizó con apoyo del Fondo Especial de Desartollo Universitario (FEDU).

\section{BIBLIOGRAFÍA}

1) Arellano P. El Libro Verde. Guía de Recursos Terapéuticos Vegetales. Ministerio de Salud. Lima, 1992. pp 33.46,53.

2) Aguilar CA. Plantas Tóxicas de México. Instituto Mejicano de Seguridad Social. México D.C., 1985.

3) Barriga R. Plantas útiles de la Amazonía peruana. CONCYTEC, Lima - Perú, 1994.

4) Sanyal AK, Das PK, Sinha S, Sinha YK. Banana and gastric secretion. J Pharm Pharmacology $1961 ; 13$ : 318-9.

5) Sanyal AK, Burnerjee CR, Das PK. Studies on peptic ulceration - Part II Role of banana in restraint and prednisolone indused ulcer in albino rals. Arch Intern Pharmacodynamie Ther 1965; 155: 244-8

6) Adami E, Marzzi-Uberti E, Turba C. Pharmacological research on gafarnate, a new synthetic isopenoid with an antiulcer action. Arch Intern Pharmacodynamie Ther 1964; 147: 113-45.

7) Tapia SB, Principe H, Rachumi M. Estudio controlado del efecto preventivo y terapéutico de Croton palanostigma, Aloe vera y Omeprazol sobre lesiones gástricas inducidas por etanol en ralas albinas. Congreso Peruano de Gastroenterología. Lima. 1996.

8) Malaga E. Erecto del clorhiurato de taspina sobre la curación de úlcera gástrica inducida $\mathrm{cn}$ ratas. Tesis de bachiller en Biología. Lima. Univ. Peruana Cayerano Heredia, 1991.

9) Robert A, Nezamis RA, Lancaster JE, Hanchar AJ. Cytoprotection by prostaglandins in rats. Prevention of gastric necrosis produced by alcohol, $\mathrm{HCl}, \mathrm{NaOH}$, hypertonic $\mathrm{NaCl}$ and thermal injury. Gastroenterology 1979; 77: 433-43.

10) Davenport IXW. Ethanol damage to canine oxyntic glandular mucosa. Proc Soc Exp Biol Med 1967; 126: 657-62.

11) Tarnawski A, Hollander K, Krause NH, Gergely H. Sucral fate protection of gastric mucosa againsl alcohol injury. Morphologic, ultraestrucural, and functional time-sequence analysis [resumen] Gastroenterology 1983; 84-113.

12) Derelanko J, Long JR. Carbenoxolone sodium protects rat gastric mucosa against ethanol induced necrosis. Proc. Soc Exp Biol Med 1981; 166: 394-7.

13) Tarnawski A, Hollander D, Gergely H, Stachura J. Comparison of Antacid, Sucralfate, Cimetidine and Ranitidine in protection of the gastrica mucosa against ethanol injury. Am J Med 1985; 79: 19-23.

14) Tarnawski A, Hollander D, Cummings D, Krause WJ, Gegerly H, Zipser RD. Are antacids acid neutralizers only Histologic uitraestructural and functional changes in normal gastric mucosa induced by antacids? [resumen]. Gastroenterology 1984; 86: 1276

15) Vaisberg AJ, Milla M, Planas MC, Cordoba JL, Agusti ER, Ferreyra R, Nustiga MC, Carlin L, Hammon GB. Taspine is cicatrizant principle in sangre de grado extracted from Croton iechleri. Planta Med 1989 Abr; 55(2): 140-3.

16) Cheng ZP, Cat $X$, Phillipson D. Studies on the antitumor: antibacterial and wound-healing properties of dragonis blood. Planta Med 1994 Dec; 60(6): 541-5.

17) Milla ME. Estudio sobre mecanismo de acción del principio acsivo taspina de sangre de grado. Tesis de bachiller en Biología. Univ. Peruana Cayetano Heredia. Lima, 1985.

18) Harrington SJ, Schelegel JF, Code CF. The protective effect os sucralfate on the gastric mucosa of rats. J Clin Gastroenterol 1981; 3: 129-34.

19) Nagashima R, Hashino E, Hinohara Y, Sakai K, Haa S, Nakano H. Effect of sucralfate on ethanol induced gastric mucosal damage in the rat. Scand I Gastroenterol 1983; 3(suppl 83): $17-20$.

20) Nagashima R. Mechanism of action of sucralfate. J Clin Gastroenterol 1981; 3(suppl 2): 117-27.

21) Nakazama $S$, Nagashima R, Samioff IM. Selective binding of sucralfate to gastric ulcer in man. Dig Dis Sci 1981; 26: 297-300.

22) Tarnawski A, Hollander D, Krause WJ, Zipser RD, Gegerly $\mathrm{H}$. Effect of sucralfate on normal gastric mucosa. Histologic. ultraestructural and functional assesment [resumen]. Gastroenterology 1983; 84; 1331 .

23) Hollander D, Tarnawski A, Krause WJ, Gegerly $H$. The protective effect of sucralface against alcohol induced gastric 
mucosal injury in the rat. Macroscopic, histologic, ultraestruciural and functional time-sequence analysis. Gastroenterology 1985; 88: 366-74

24) Tarnawski A, Hollander D. Prostaglandin protection of the rat gastric mucosa against alcohol injury a mucosal proliferative zone. Gastroenterology 1985; 88: 334-52.

25) Tarnawski A, Hollander D. Arachidonic acid protection of gastric mucosa against alcohol injury sequencial analysis of morphologic and functional changes. J Lab Clin Med 1983; 102: 340-51.

26) Tarnawski A, Hollander D, Mach T, Stachura J, Bogdal J. Effect of sucralfate on normal human gastric mucosa. Endoscopic, histologic and uitraestructural assesment [resumen] Gastroint Endosc 1984; 30: 155

27) Mylake $T$, Ariyoshi J, Suzaki T, Oishi M, Sakai M, Ueda S. Endoscopic evaluation of the effect of sucralfate therapy and other clinical parameters on the recurrence rate of gastric ulcer. Dig Dis Sci 1980; 25: 1-7.

28) Moshal MG, Spitaeis JM, Manion GI. Double-blind, placebocontrolled evaluation of one-year therapy with sucralfate in healed duodenal ulcer. Scand $J$ Gastroenterol 1982; 18(suppl 83): $57-9$

29) Classen M, Bethge $H$, Brunner G, et al. Effect of sucralfate on peptic ulcer recurrence: a controlled, double-blind multicenter study. Scand J Gastroenterol 1982; 18(suppl 83): 61-8.

30) Libeskind $M$. Maintenance treatment of patients with healed peptic uicer with sucralfate, placebo, and cimetidine. Scand J Gastroenterol 1982; 18(suppl 83): 69-70.

31) Borrego E, Margolis J, Bank S, Schulman N, Chardavoyne R. A comparison between sucralfate and antacids in the prevention of stress uicers in critically ill patients [resumen]. Gastroenterology 1984; 86: 102. 